\title{
Is a Novel Nomogram Better Than TNM Staging at Predicting Survival in Siewert Type 2 Adenocarcinoma?
}

\author{
Kimberly J. Song, $\mathrm{MD}^{1}$ and Andrew T. Arndt, $\mathrm{MD}^{2}$ \\ ${ }^{1}$ Department of Cardiothoracic Surgery, NYU Langone Health, New York, NY; ${ }^{2}$ Department of Cardiovascular and \\ Thoracic Surgery, Rush University Medical Center, Chicago, IL
}

The addition of radiation to the chemotherapeutic treatment of esophageal adenocarcinoma has become increasingly common, with evidence to support an improved complete response rate ${ }^{1}$ and decreased systemic toxicity. ${ }^{2}$ In their article titled "Proposal of a nomogram for predicting survival in patients with Siewert type II adenocarcinoma of esophagogastric junction after preoperative radiation", Liu et al. present a scoring system for determining postoperative pathologic predictors of overall survival.

This retrospective analysis utilized data from the Surveillance, Epidemiology, and End Results (SEER) database from 1998 to 2013, yielding a cohort of 1818 patients with M0 disease who had preoperative radiation, surgical treatment, and pathologic examination of lymph nodes. Three lymph node elements were assessed: positive lymph node (PLN) count, lymph node ratio (LNR; ratio of positive nodes vs. total nodes), and log odds of positive nodes $\quad$ LOODDS; $\log _{\mathrm{e}}$ ([PLN + 0.5]/[negative $\mathrm{LN}+$ $0.5])\}$. While univariate analysis identified age, sex, tumor grade, histology type, and all three nodal elements as significant prognostic factors, only age, tumor grade, PLN count, and LODDS value were deemed significant on multivariate analysis. Notably, ypT classification was not statistically significant in either univariate or multivariate analysis.

Derivation and validation sets were used to generate a nomogram with point values assigned to each predictor, the most significant of which was LODDS value. Other

\footnotetext{
(C) Society of Surgical Oncology 2019

First Received: 25 January 2019;

Published Online: 15 February 2019

K. J. Song, MD

e-mail: kimberlysong@gmail.com
}

significant predictors included PLN number, age, and histology. The total score was used to determine the 2-, 3-, and 5-year survival probabilities, and the authors validated the nomogram using a calibration plot of the model. When comparing it with TNM staging using decision curve analyses and the Harrell's C-index, the authors found the nomogram to have higher prognostic value.

This study is valuable in its specific analysis of Siewert type II adenocarcinomas, as well as in its focus on pathologic variables for predicting overall survival, although there are limitations. As the authors themselves mention, information regarding tumor status prior to radiotherapy was unavailable, limiting the ability to assess clinical response to neoadjuvant therapy. Since pathologic complete response has been associated with increased overall and disease-free survival, ${ }^{3}$ this information would be useful.

Information regarding surgical margin status and chemotherapy was also unavailable. The time interval between neoadjuvant therapy and surgical intervention may also be relevant as longer delays to resection may contribute to decreased $30-$ day $^{4}$ or overall survival. ${ }^{5}$ Finally, the quantity of lymph nodes analyzed was subdivided into only two categories (1-15 nodes vs. $>16$ nodes), which may overlook subtleties in the predictive ability of the nomogram. Without this information, it may be difficult to know exactly which patient groups can be included in this nomogram.

This manuscript provides the basis for a systematic approach to analyzing our pathologic findings after preoperative radiotherapy and esophagectomy for Siewert type II adenocarcinomas. Analyzing the unique characteristics of this cohort is appropriate since different Siewert classifications have been shown to behave differently, with more distal disease associated with larger tumors, more aggressive behavior, and decreased overall survival. ${ }^{6}$ 
Ultimately, while this study may not definitively pave the way for an overhaul of gastroesophageal tumor staging, it charges us to continue to improve our prognostication methods. With ongoing validation and refining, we may be able to broadly apply a nomogram such as this, enhancing our ability to provide a more specific and relevant survival estimate to patients with this disease.

\section{REFERENCES}

1. Tiesi G, Park W, Gunder M, et al. Long-term survival based on pathologic response to neoadjuvant therapy in esophageal cancer. $J$ Surg Res. 2017;216:65-72.

2. Anderegg MCJ, van der Sluis PC, Ruurda JP, et al. Preoperative chemoradiotherapy versus perioperative chemotherapy for patients with resectable esophageal or gastroesophageal junction adenocarcinoma. Ann Surg Oncol. 2017;24(8):2282-2290.
3. Soror T, Kho G, Zhao KL, Ismail M, Badakhshi H. Impact of pathological complete response following neoadjuvant chemoradiotherapy in esophageal cancer. J Thoracic Dis. 2018;10(7):4069-4076.

4. van der Werf LR, Dikken JL, van der Willik EM, van Berge Henegouwen MI, Nieuwenhuijzen GAP, Wijnhoven BPL. Time interval between neoadjuvant chemoradiotherapy and surgery for oesophageal or junctional cancer: a nationwide study. Eur $J$ Cancer. 2018;91:76-85.

5. Qin Q, Xu H, Liu J, et al. Does timing of esophagectomy following neoadjuvant chemoradiation affect outcomes? A meta-analysis. Int J Surg. 2018;59:11-18.

6. Curtis NJ, Noble F, Bailey IS, Kelly JJ, Byrne JP, Underwood TJ. The relevance of the Siewert classification in the era of multimodal therapy for adenocarcinoma of the gastro-oesophageal junction. $J$ Surg Oncol. 2014;109(3):202-207.

Publisher's Note Springer Nature remains neutral with regard to jurisdictional claims in published maps and institutional affiliations. 